\title{
UV-induced photochemistry of matrix-isolated 1-phenyl-4-allyl-tetrazolone $\dagger$
}

\author{
L. M. T. Frija, ${ }^{a, b}$ I. D. Reva, ${ }^{b}$ A. Gómez-Zavaglia, ${ }^{b, c}$ M. L. S. Cristiano ${ }^{a}$ and R. Fausto ${ }^{* b}$ \\ Received 15th March 2007, Accepted 20th June 2007 \\ First published as an Advance Article on the web 11th July 2007 \\ DOI: 10.1039/b703961a
}

The photochemistry and molecular structure of 1-phenyl-4-allyl-tetrazolone (PAT) was studied by FT-IR matrix isolation spectroscopy and DFT(B3LYP)/6-311++G(d,p) calculations. The spectrum of matrix-isolated PAT monomers agrees well with the sum spectrum of three conformers predicted theoretically. UV irradiation $(\lambda>235 \mathrm{~nm})$ of matrix-isolated PAT induces three types of photofragmentation: (1) production of phenylazide and allyl-isocyanate, with phenylazide then losing $\mathrm{N}_{2}$ to yield 1-aza-1,2,4,6-cycloheptatetraene; (2) formation of phenyl-isocyanate and allylazide; (3) $\mathrm{N}_{2}$ elimination leading to formation of 1-allyl-2-phenyldiaziridin-3-one; this compound partially reacts further to form 1-allyl- $1 H$-benzoimidazol-2(3H)-one. The observed photochemistry of the matrix-isolated PAT is distinct from the preferred photochemical fragmentation in solution, where 3,4-dihydro-3-phenylpyrimidin-2(1H)-one is produced as the primary photoproduct.

\section{Introduction}

The application of tetrazoles in fields of great relevance, such as medicine or agriculture, is widespread and well-known. In many drugs and drug-candidates, replacement of carboxylic acid groups $-\mathrm{CO}_{2} \mathrm{H}$ by tetrazolic acid fragments $\mathrm{CN}_{4} \mathrm{H}$ is frequent. Despite the similarities in physical characteristics of these two groups, the tetrazolic acid fragment is the preferred one in several cases due to its superior metabolic stability at physiologic $\mathrm{pH}$, and replacement of $-\mathrm{CO}_{2} \mathrm{H}$ by $-\mathrm{CN}_{4} \mathrm{H}$ typically results in an increase in bioavailability of the pharmacophore. For instance, the tetrazolic ring has been used to modify the structure of the heme environment in myoglobin through incorporation of the $\mathrm{N}$ tetrazol-5-yl histidine unit, ${ }^{1} 3^{\prime}$-tetrazolo-3'-deoxythymidines were the first approved drugs for AIDS treatment and several tetrazole derivatives have also been explored for antituberculotic activity. ${ }^{2,3}$ 5-Oxo-substituted tetrazoles, i.e., tetrazolones are particularly stable metabolically. Tetrazolone derivatives have been used as potent and selective agonists of the $\beta 3$ human adrenergic receptors ( $\beta 3$-HAR). The receptor is linked to the increase in metabolic rates in humans and, as a result, $\beta 3$-HAR agonists are useful in the treatment of obesity. Previously developed, $\beta 3$-HAR agonists containing imidazolidinone and imidazolone rings were found to have quite low oral bioavailability due to their extensive metabolism. The higher stability of tetrazolone derivatives allowed to overcome this problem. ${ }^{4-6}$

Tetrazoles also have many other applications, such as in agriculture, ${ }^{7}$ photography and photoimaging ${ }^{8}$ and in automobile industry as gas generating agents for airbags. ${ }^{9}$

${ }^{a}$ Department of Chemistry and Biochemistry, F.C.T., and CCMAR, University of Algarve, Campus de Gambelas, 8005-039, Faro, Portugal

${ }^{b}$ Department of Chemistry, University of Coimbra, Coimbra, P-3004-535, Portugal.E-mail: rfausto@ci.uc.pt; Fax:+351239827 703

'Faculty of Pharmacy and Biochemistry, University of Buenos Aires, C.P. 1113, Buenos Aires, Argentina

$\dagger$ Electronic supplementary information (ESI) available: Theoretical structural and vibrational data for the three conformers of 1-phenyl-4-allyltetrazolone. See DOI: 10.1039/b703961a
In view of the scientific and industrial relevance of tetrazolyl derivatives, our research program has long been focused on the investigation of structure-reactivity relationships in tetrazolebased compounds. Regarding their photoreactivity, it has been shown that tetrazole-ring cleavages, leading either to azides or aziridines, represent the main characteristic photochemical reactions of this family of heterocycles. However, the structure of the starting compound and the reaction conditions strongly determine the precise nature and relative amounts of the final photoproducts, making this type of compounds a permanent challenge to research. ${ }^{10,11}$

In recent contributions we have described the photochemistry of some representative tetrazolyl compounds isolated in lowtemperature matrices. ${ }^{12-15}$ The photochemistry of a series of 4-allyltetrazolones in solution ${ }^{16}$ was also addressed, and the investigation has led to a new synthetic strategy for the one-step and high yielding photochemical conversion of the 1-substituted-4-allyltetrazol-5-ones into 3,4-dihydro-pyrimidinones, an important class of heterocycles. ${ }^{17}$ Thus, 1,4-substituted-5-tetrazolones are especially versatile as intermediate compounds in the synthesis of other heterocyclic systems. Besides the already mentioned relevance of tetrazoles, compounds bearing an allylic alcohol function are often vital structural units of biologically active systems, and also act as key intermediates in synthesis. ${ }^{18,19}$ 4-Allyl-tetrazolones are readily obtained from reaction of an allylic alcohol and 5-chloro tetrazole. ${ }^{20,21}$

Based on the above expressed considerations, we hereby describe the results of our recent investigation on the structure and reactivity of 4-allyl-tetrazolyl derivatives. 1-Phenyl-4-(prop-2-enyl)tetrazol-5-one (1-phenyl-4-allyl-tetra-zolone, PAT) was selected as the subject of the present study. The structure of PAT monomers isolated in low temperature inert gas matrices as well as their UV induced photochemistry were studied by means of infrared spectroscopy, supported by extensive DFT calculations.

The matrix isolation technique coupled to a suitable probing method such as FT-IR spectroscopy represents a powerful approach to improve our understanding of the photochemistry of tetrazole derivatives. In the matrix, the processes are cage-confined 
and molecular diffusion is inhibited, thereby introducing a useful simplification for the investigation of the reaction mechanisms involved. The main advantage of the matrix-isolation technique is the possibility to achieve a considerably high spectroscopic resolution, increasing the probability to detect chemical species produced in low amounts during photolysis or those with intrinsically low intensity IR absorptions. Furthermore, due to the high resolution of the method, it is possible to distinguish spectral signatures of different tautomers or conformers, usually closely located in the spectrum, and it becomes possible to compare directly the experimental spectra with the results of theoretical calculations. The low temperature conditions can be used for trapping and spectroscopic identification of, at least, some of the intermediate transient products, which otherwise are very reactive.

\section{Experimental methods}

1-Phenyl-4-(prop-2-enyl)-tetrazol-5-one (1-phenyl-4-allyl-tetrazolone, PAT) was synthesised as described elsewhere. ${ }^{16}$ The IR spectra of PAT were obtained using a Mattson (Infinity 60AR Series) Fourier transform infrared spectrometer, equipped with a deuterated triglycine sulfate (DTGS) detector and a $\mathrm{Ge} / \mathrm{KBr}$ beamsplitter, with $0.5 \mathrm{~cm}^{-1}$ spectral resolution. In order to avoid interference from atmospheric $\mathrm{H}_{2} \mathrm{O}$ and $\mathrm{CO}_{2}$, a stream of dry nitrogen continuously purged the optical path of the spectrometer. The compound was placed in a specially designed doubly thermostattable Knudsen cell, ${ }^{22}$ whose compartments (sample container and valve nozzle) were kept at $350 \mathrm{~K}$ during deposition of the matrix. Matrices were prepared by co-deposition onto the CsI substrate of the cryostat cooled to $10 \mathrm{~K}$, of PAT vapors coming out of the Knudsen cell and a large excess of the matrix gas (argon N60, Air Liquide) coming from a separate line. The obtained matrices did not present any evidence for the presence of associates of the deposited compound. All experiments were performed using an APD Cryogenics closed-cycle helium refrigeration system with a DE-202A expander.

Irradiation of the samples was carried out with a $150 \mathrm{~W}$ xenon arc lamp (Osram XBO $150 \mathrm{~W} / \mathrm{CR}$ OFR), through the outer $\mathrm{KBr}$ window of the cryostat $(\lambda>235 \mathrm{~nm})$.

\section{Theoretical calculations}

The quantum chemical calculations of the stationary points were performed at the DFT level of theory using the standard $6-311++\mathrm{G}(\mathrm{d}, \mathrm{p})$ basis set and the three-parameter density functional abbreviated as B3LYP, which includes Becke's gradient exchange correction $^{23}$ and the Lee, Yang, Parr correlation functional. ${ }^{24}$ Potential energy scans were performed using the 6-31G $(\mathrm{d}, \mathrm{p})$ basis set.

Geometrical parameters were optimized using the Geometry Direct Inversion of the Invariant Subspace (GDIIS) method. ${ }^{25}$ Optimization of geometries was followed by calculation of the vibrational spectra at the same theory level. The nature of the obtained stationary points was checked through the analysis of the corresponding Hessian matrix. A set of internal coordinates was defined, and the Cartesian force constants were transformed to the internal coordinate space, allowing ordinary normalcoordinate analysis as described by Schachtschneider, ${ }^{26}$ by using the optimized geometries and harmonic force constants resulting from the DFT(B3LYP)/6-311++G(d,p) calculations.

The calculated harmonic frequencies (scaled with a factor of $0.978)$ t: were used to assist the analysis of the experimental spectra and to account for the zero-point vibrational energy (ZPVE) corrections. All calculations in this work were carried out using the Gaussian 03 program. ${ }^{27}$

\section{Results and discussion}

The geometry optimisations carried out for 1-phenyl-4-allyltetrazolone (PAT), led to the identification of three low energy local minima, corresponding to the structures Sk' ("Skew-minus"), Sk ("Skew-plus") and Syn, represented as Newman projections in Fig. 1 (for optimized geometries see the electronic supplementary information (ESI)). $\uparrow$ All these minima depend on the position of the ethene group on the allylic chain attached to the tetrazole ring, and their structures result from rotation of this group around the $\mathrm{C}_{(7)}-\mathrm{C}_{(8)}$ bond in PAT. The potential energy profile for this internal rotation (Fig. 1) was calculated by incrementing the value of the $\mathrm{N}_{(4)}-\mathrm{C}_{(7)}-\mathrm{C}_{(8)}=\mathrm{C}_{(9)}$ dihedral angle and fully optimizing all other geometric parameters. The three low energy conformers $\mathrm{Sk}^{\prime}$, Sk and Syn have very close relative energies, which fall in the $0-2.3 \mathrm{~kJ} \mathrm{~mol}^{-1}$ range (see Fig. 1). At the B3LYP/6-311++G(d,p) level, the relative energies of $\mathrm{Sk}^{\prime}$, Sk and Syn were calculated to be $0,0.2$ and $2.2 \mathrm{~kJ} \mathrm{~mol}^{-1}$, respectively (ZPVE corrections included). The small differences in the energy of conformers can be explained considering interactions between the allylic chain and the tetrazole ring of PAT. In conformer $\mathrm{Sk}^{\prime}$ (the lowest energy structure) the ethene group on the allylic chain is as far as possible from the tetrazole ring and opposite to the carbonyl oxygen $\mathrm{O}_{(6)}$, which minimises repulsive interactions in the molecule and consequently its energy. Also, in conformer Sk the ethene group on the allylic

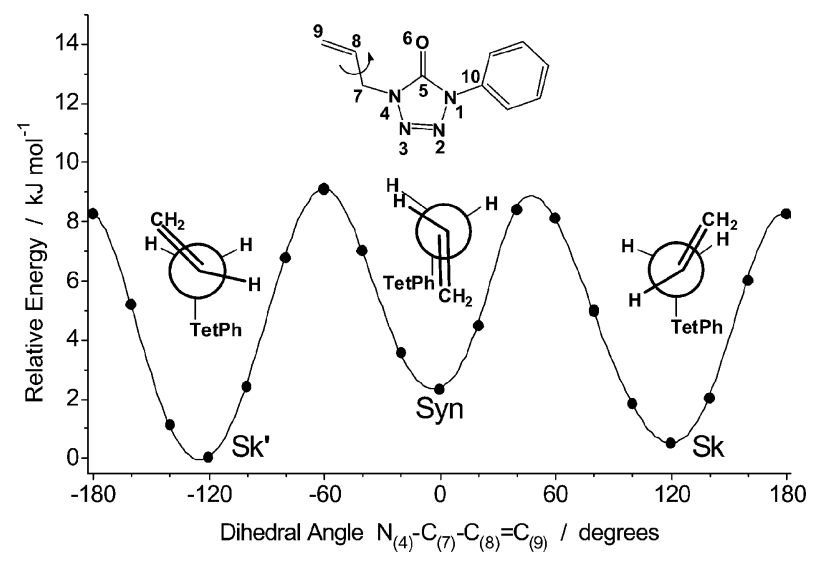

Fig. 1 Relaxed potential energy scan for internal rotation of the ethene group around the $\mathrm{C}_{(7)}-\mathrm{C}_{(8)}$ bond in 1-phenyl-4-allyl-tetrazolone, calculated at the DFT(B3LYP)/6-31(d,p) level of theory. The reaction coordinate was incrementally fixed while all other geometrical parameters were fully optimized. Minima denoted as $\mathrm{Sk}^{\prime}$, Syn and $\mathrm{Sk}$ correspond to conformers "Skew-minus", Syn, "Skew-plus" and are represented as Newman projections. The lowest-energy structure is conformer $\mathrm{Sk}^{\prime}$. Its energy ( -681.271842 hartrees) was chosen as the relative zero level.

$\$$ This scaling factor has been found to provide a good match between calculated and observed spectra of several tetrazole-substituted compounds we investigated previously. ${ }^{12-15}$ 
chain remains distant from the tetrazole ring, but the closer proximity of the ethene group to the oxygen atom is responsible for a slight increase in energy of Sk relatively to the $\mathrm{Sk}^{\prime}$ form $(\Delta E=$ $0.2 \mathrm{~kJ} \mathrm{~mol}^{-1}$ ). The structure Syn is the most unstable conformer among the three calculated minima, this fact being attributed to the spatial proximity between the ethene group and the tetrazole ring.

Calculations show that in all conformers the phenyl and tetrazole rings have approximately planar geometries and are coplanar to each other. This last result can be mostly attributed to the favourable interactions between the phenyl hydrogen atoms ortho to the tetrazole ring and the carbonyl oxygen and $\mathrm{N}_{(2)}$ atoms (Fig. 1). Structural data for the co-planar phenyl and tetrazolyl rings in PAT are similar to those observed for 1-phenyltetrazole5-one. ${ }^{12}$ On the other hand, also for all conformers, the geometry around the $\mathrm{N}_{(4)}-\mathrm{C}_{(7)}$ bond has been found to be the same, with the $\mathrm{C}_{(8)} \mathrm{H}=\mathrm{C}_{(9)} \mathrm{H}_{2}$ fragment being perpendicular to the tetrazolone ring.

Because of the small energy differences between the three forms, it can be expected that all of them are present in the gas phase in significant amounts. Indeed, at $77^{\circ} \mathrm{C}$, the predicted populations of forms $\mathrm{Sk}^{\prime}$, Sk and Syn are $41.8 \%, 38.8 \%$ and $19.4 \%$, respectively. The energy barriers separating these forms were predicted to be higher than $8 \mathrm{~kJ} \mathrm{~mol}^{-1}$ in both directions (calculated at the DFT(B3LYP)/6-31G(d,p) level of theory, see Fig. 1). Although these barriers are not very high, they are related with rotation of a bulky $-\mathrm{CH}_{2}-\mathrm{CH}=\mathrm{CH}_{2}$ group. The size of the group hinders its internal rotation in the rigid environment (lowtemperature matrix) and precludes interconversions between the three conformers. This implies that the relative populations of the three conformers of PAT trapped into an Ar matrix should be the same as they were in the gas phase prior to deposition. ${ }^{28}$
The experimental IR spectrum of PAT monomers, isolated in an Ar matrix, is presented in Fig. 2 (trace a). This spectrum is well reproduced by the spectrum of a conformer mixture simulated theoretically [at the DFT(B3LYP)/6-311++G(d,p) level, see Fig. 2 (trace b)]. The calculated spectra of the three forms of PAT are very similar to each other (see the electronic supplementary information (ESI)), $\dagger$ making very difficult to distinguish these forms spectroscopically. Luckily, the precise knowledge concerning the conformational orientation of the allyl group $\left(-\mathrm{CH}_{2}-\right.$ $\mathrm{CH}=\mathrm{CH}_{2}$ ) is not essential for the interpretation of photochemical transformations of PAT isolated in an argon matrix, as it will be shown below.

As mentioned above, the photochemistry in solution of a series of 4-allyl-tetrazolones, including PAT, was recently studied in our laboratory, revealing that the photolysis in alcoholic solutions leads to new heterocycles, namely 3,4-dihydro-pyrimidin-2 $(1 H)$ ones, in almost quantitative yields. In the specific case of PAT photolysis led to formation of 3,4-dihydro-3-phenylpyrimidin2(1H)-one (PDP, Fig. 3). Results from a previous investigation carried out by Quast and co-workers, under different experimental conditions, indicated that photolysis of PAT in solution leads to formation of 1-allyl-1 $H$-benzoimidazol-2(3H)-one (ABZ, Fig. 3) as the sole product. ${ }^{29}$ Both primary photoproducts, PDP and ABZ, detected upon photolysis of PAT in solution, result from elimination of nitrogen from the tetrazole ring, followed by two distinct processes of cyclization. The different reactivity revealed in these two experiments can be attributed to the different methodologies used, which clearly affect the reactivity and stability of the intermediate species involved. The divergent results obtained for the photolysis of PAT in solution made the photochemical investigation of this compound in cryogenic matrices an especially attractive challenge.

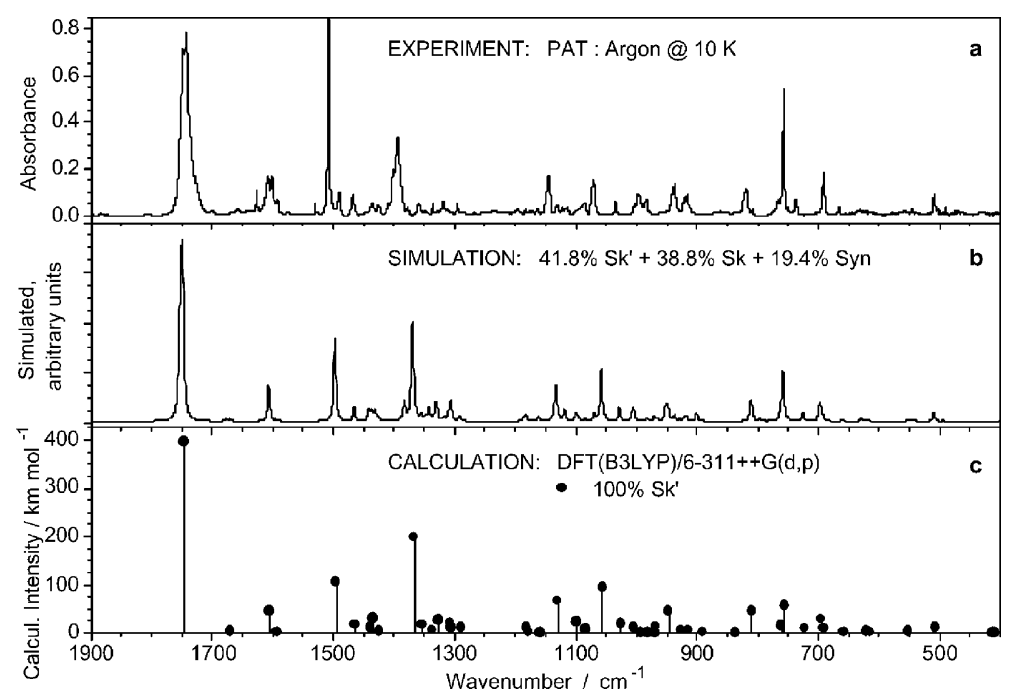

Fig. 2 (a) Experimental FTIR spectrum of monomeric 1-phenyl-4-allyl-tetrazolone (PAT) trapped in an argon matrix at $10 \mathrm{~K}$ immediately after deposition; (b) infrared sum spectrum of the characteristic equilibrium mixture of PAT conformers at $77^{\circ} \mathrm{C}$. The spectra of individual conformers were simulated with Lorentzian functions centered at the calculated (scaled) frequencies and with bandwidths-at-half-height equal to $4 \mathrm{~cm}^{-1}$. The calculated intensities of different conformers were population-weighted; (c) DFT(B3LYP)/6-311++G(d,p) calculated infrared spectrum of the most stable conformer $\left(\mathrm{Sk}^{\prime}\right)$. The calculated wavenumbers were scaled with a uniform factor of 0.978 . The calculated intensities were not scaled. For simplicity, only the spectrum of the most stable form is shown, since the predicted spectra of all conformers are very similar. Spectra of individual conformers are provided in the electronic supplementary information (ESI) $\dagger$ 


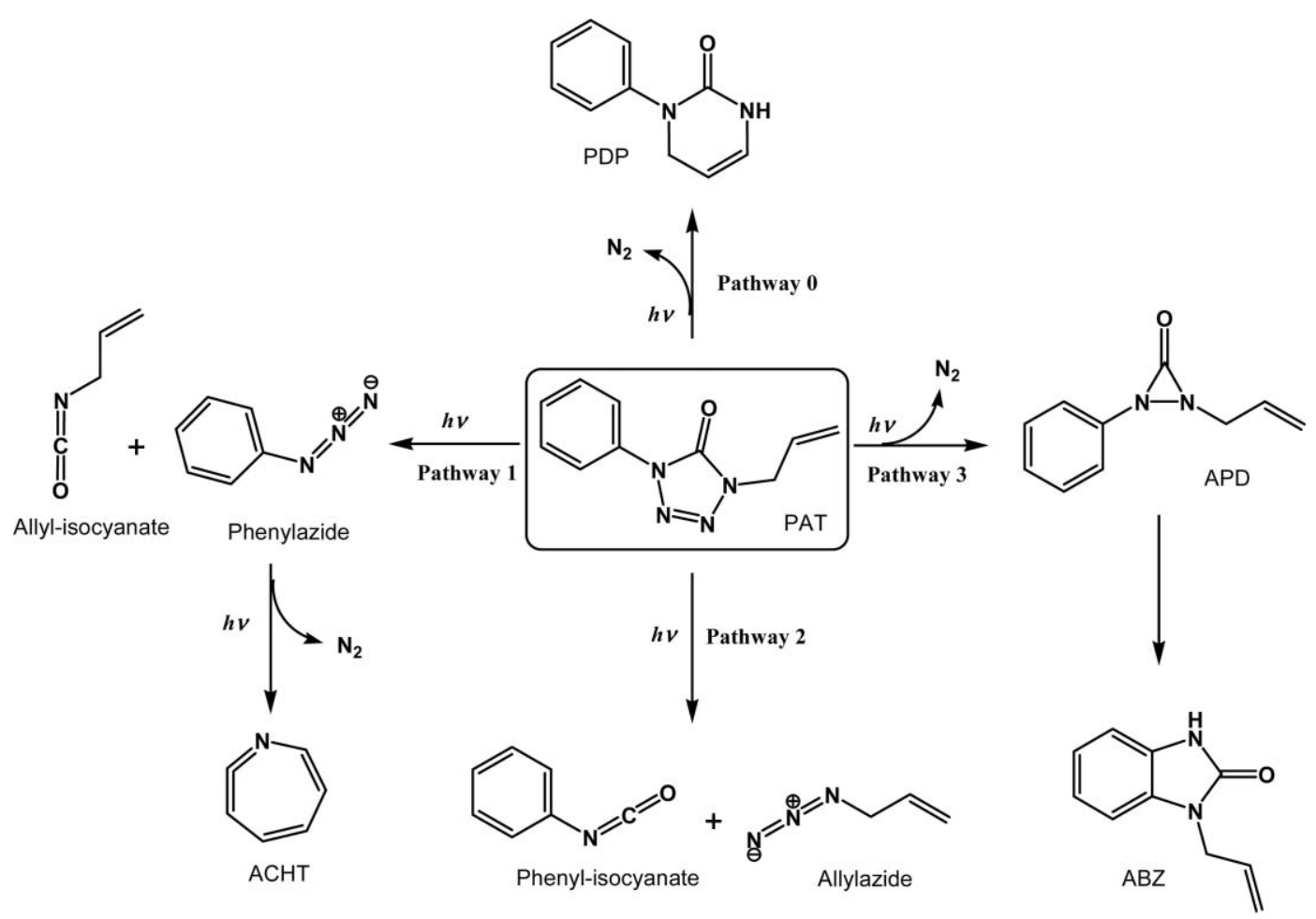

Fig. 3 Proposed photochemical pathways resulting from irradiation of 1-phenyl-4-allyl-tetrazolone (PAT) in an argon matrix through the outer KBr window of the cryostat $(\lambda>235 \mathrm{~nm})$.

To study the photochemistry of the matrix-isolated PAT, in situ UV-irradiation $(\lambda>235 \mathrm{~nm})$ experiments were carried out. The resulting photochemical processes were followed by IR spectroscopy and the interpretation of the experimental spectroscopic data was supported by extensive DFT calculations on plausible photoproducts, taking into consideration previously reported spectroscopic data, whenever available.

Upon UV irradiation of matrix-isolated PAT, the intensities of the bands of this compound decreased significantly while new bands appeared in the infrared spectrum, indicating that the compound was transformed in diverse photoproducts. The changes are particularly evident in the $2300-1700 \mathrm{~cm}^{-1}$ spectral region, which is shown in Fig. 4(A). For the conformationally flexible photoproducts all conformational possibilities were explored (about one week of parallel calculations on several PCs). In the simulated spectra, only the most stable conformers for each photoproduct were considered (Fig. 4). Further conformers were neglected in this analysis on the basis that their calculated spectra are very similar within the same photoproduct. The validity of such approach is demonstrated by a reasonable correspondence between the simulated and the observed spectra of photoproducts.

Photochemically induced cleavage of the tetrazole ring has been found to be a common process. ${ }^{10,11,29-33}$ As mentioned before, tetrazole itself easily eliminates molecular nitrogen to produce nitrilimine, which then undergoes secondary photoreactions, such as isomerization to carbodiimide, cyanamide or a hydrogen cyanidenitrene complex. ${ }^{10}$ The proposed reaction pathways resulting from irradiation of PAT are schematically shown in Fig. 3.

The photochemistry of the matrix-isolated compound reveals three main reaction pathways: (1) cleavage of the tetrazole ring through the formally single bonds $\mathrm{C}_{(5)}-\mathrm{N}_{(1)}$ and $\mathrm{N}_{(3)}-\mathrm{N}_{(4)}$, with production of phenylazide and allyl-isocyanate as primary photoproducts (phenylazide can then undergo additional reactions to give 1-aza-1,2,4,6-cycloheptatetraene); (2) cleavage of the $\mathrm{N}_{(1)}-\mathrm{N}_{(2)}$ and $\mathrm{C}_{(5)}-\mathrm{N}_{(4)}$ bonds, leading to formation of phenyl-isocyanate and allylazide, and (3) cleavage of the $\mathrm{N}_{(1)}-\mathrm{N}_{(2)}$ and $\mathrm{N}_{(3)}-\mathrm{N}_{(4)}$ bonds producing 1-allyl-2-phenyldiaziridin-3-one (APD). This compound is then partially transformed in 1-allyl-1 $H$-benzoimidazol-2(3H)one (ABZ).

The possible formation of PDP from PAT, shown by the Pathway "0" in Fig. 3, was found not to occur under the present experimental conditions, contrarily to what was observed during the photolysis of PAT in solution. The designation of Pathway "0" illustrates that the outcome of this channel in the argon matrix at $10 \mathrm{~K}$ is equal to $0 \%$. The non-formation of PDP in this study is doubtlessly related to the matrix medium where the reaction is suppressed. We believe that a strong restriction of conformational mobility in the argon matrix affects not only PAT itself (see above), but also its photoproducts. The design of PDP from PAT (after extrusion of $\mathrm{N}_{2}$ ) involves an approach between the terminal carbon of the allylic chain and the nitrogen atom $\mathrm{N}_{(1)}$, in order to form a new $\mathrm{C}-\mathrm{N}$ single bond. Such approach is strongly hindered in the rigid matrix, which consequently precludes formation of PDP. The inclusion of the hypothetic reaction channel leading to PDP in the general scheme presented in Fig. 3 results from the initial assumption that this could be a possibility, in view of the results obtained in solution. For the sake of simplicity, the calculated spectrum of PDP is omitted in part B of Fig. 4.

The observed photoprocesses in the argon matrix (Pathways 1 to 3 in Fig. 3) result from cleavage of the four formally single bonds in different combinations, whereas every primary photoreaction 

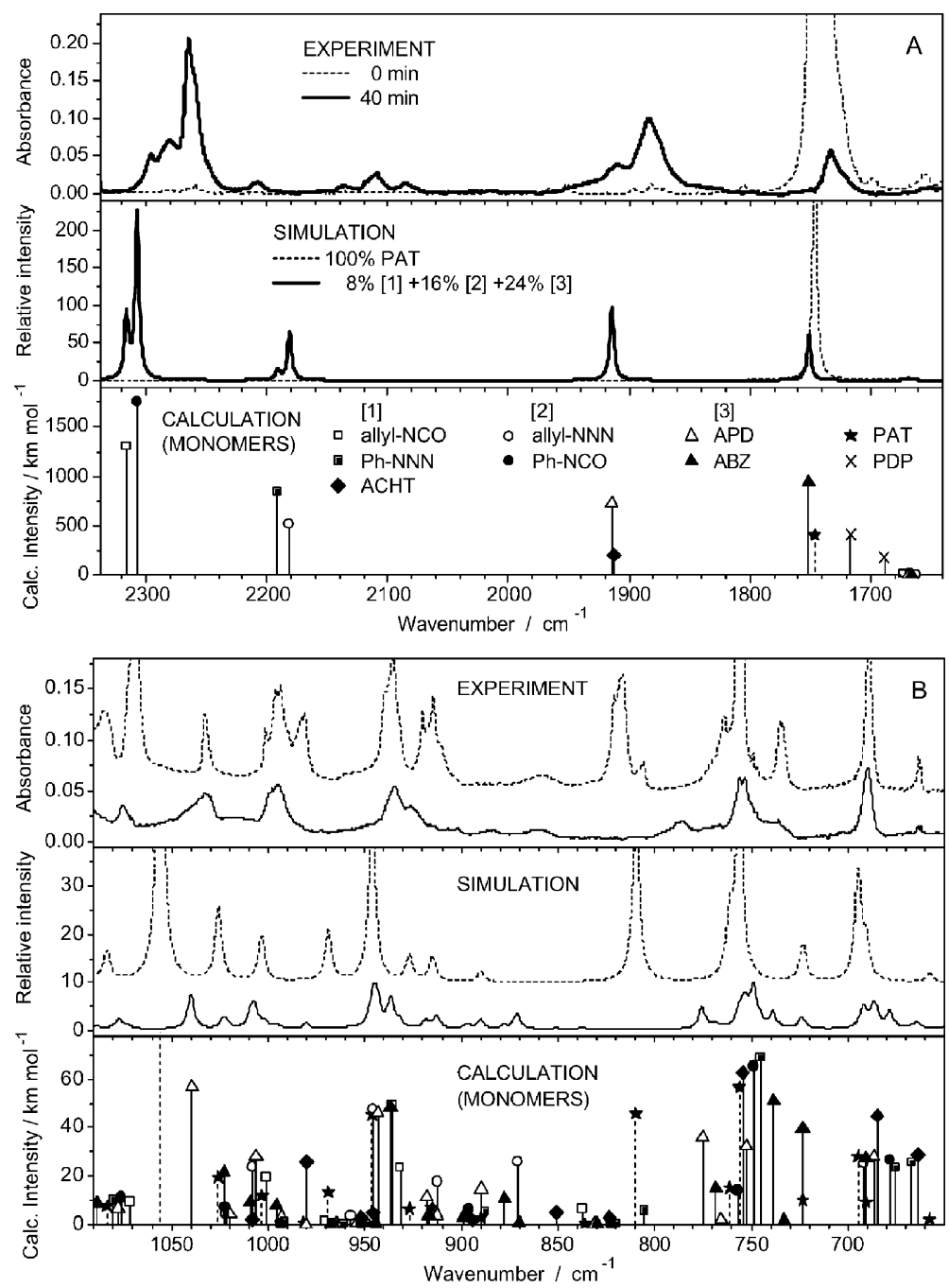

Fig. 4 Infrared spectra of 1-phenyl-4-allyl-tetrazolone (PAT) and the proposed photoproducts, in the carbonyl stretching (A) and fingerprint (B) regions. Upper frames (EXPERIMENT): dashed line-FTIR spectrum of monomeric PAT trapped in an argon matrix at $10 \mathrm{~K}$ immediately after deposition ( $0 \mathrm{~min}$ of irradiation); solid line - extracted spectrum of the photoproducts formed after 40 min of UV-irradiation $(\lambda>235 \mathrm{~nm})$ of PAT trapped in an Ar matrix. The extracted spectrum was obtained by subtraction of the scaled spectrum of non-irradiated matrix from the spectrum of irradiated sample. The scaling factor was chosen so that the absorptions due to the originally deposited compound (PAT) were nullified. Lower frames (CALCULATION): DFT(B3LYP)/6-311++G(d,p) calculated spectra of PAT (dashed sticks) and possible photoproducts. The calculated wavenumbers were scaled with a uniform factor of 0.978 . The calculated intensities were not scaled. Groups [1], [2] and [3] designate different photochannels. Middle frames (SIMULATION): simulated infrared spectra of PAT (dashed line) and a mixture of photoproducts (solid line). They were created using Lorentzian functions centered at the calculated (scaled) frequencies (shown in the lower frames) and with bandwidths-at-half-height equal to $4 \mathrm{~cm}^{-1}$. The calculated intensities in the spectrum of PAT were taken as $100 \%$. The calculated intensities of photoproducts were scaled down to reproduce the relative intensities in the experimental spectrum of photoproducts in the carbonyl stretching region (part A) and to obey the normalization condition: total amount of photoproducts is equal to the amount of consumed reagent (48\%). Note that in the fingerprint region (part B), the experimental (upper frame) and simulated (middle frame) spectra of PAT (dashed lines) are shifted on ordinate for clarity. The symbols of PAT and photoproducts are the same in parts $\mathrm{A}$ and $\mathrm{B}$.

involves cleavage of two single bonds in the tetrazole ring. After 40 min of UV-irradiation, the amount of the consumed reagent is equal to $48 \%$. This percentage should then correspond to the sum of efficiencies of the three reaction pathways and to the total amount of photoproducts.

All strong and medium intensity bands calculated for the two primary photoproducts formed in Pathway 1 (phenylazide and allyl-isocyanate) could be observed in the spectra of the irradiated matrix (see Fig. 4). The experimental spectrum of aliphatic isocyanates, such as allyl-isocyanate, is usually easily identifiable, because it shows an intense vibration in the $2290-2300 \mathrm{~cm}^{-1}$ region due to the $v \mathrm{~N}=\mathrm{C}=\mathrm{O}$ antisymmetric stretching vibration. In the spectrum of photoproducts (Fig. 4(A)) the band associated to this vibration emerges at $2296.5 \mathrm{~cm}^{-1}$, proving formation of allylisocyanate upon PAT photolysis.

Pathway 1 shows a secondary process resulting from conversion of phenylazide in 1-aza-1,2,4,6-cycloheptatetraene (ACHT). Phenylazide has been previously isolated and irradiated in an argon matrix, and its conversion into ACHT clearly demonstrated. ${ }^{12}$ The vibrational spectra of matrix isolated phenylazide ${ }^{34}$ and of 
ACHT are well known and their identification was made without any difficulty. In the spectrum of the photoproducts, the most prominent band of phenylazide appears at $2108.8 \mathrm{~cm}^{-1}$ and is ascribed to the $v \mathrm{~N}=\mathrm{N}^{+}=\mathrm{N}^{-}$antisymmetric stretching vibration. Characteristic bands of $\mathrm{ACHT}^{12}$ were also clearly identified in the spectra of the irradiated matrix. Particularly evident is the band-mark associated with the intense antisymmetric stretching vibration of the ketenimine moiety $(v \mathrm{C}=\mathrm{C}=\mathrm{N}$ as.) of ACHT, observed at $1910.5 \mathrm{~cm}^{-1}$ (Fig. 4).

The efficiencies of the different photochannels were estimated on the basis of the comparison between the relative band intensities for the photoproducts observed experimentally. These intensities were pre-normalized by the calculated intensities of the putative photoproducts, using the observed and calculated absorptions in the 1600-2400 $\mathrm{cm}^{-1}$ spectral region (see Fig. 4(A)). According to the available literature data, the average absolute difference in the intensities calculated using the applied theoretical method and basis set can be estimated to be $c a$. $6 \mathrm{~km} \mathrm{~mol}^{-1}$, the method showing a performance in close agreement with the highlevel QCISD $a b$ initio method. ${ }^{35}$ All the photoproduct bands in the used spectral range have absolute calculated intensities in the range $200-1500 \mathrm{~km} \mathrm{~mol}^{-1}$ (see Fig. 4(A)). This leads to expected uncertainties below $3 \%$ in the calculated frequencies. A subsequent normalization was carried out in order to bring the total efficiency of all photochannels to be equal to the amount of consumed reagent (i.e. $48 \%$ after $40 \mathrm{~min}$ ). The validity of this normalization procedure was then confirmed in the fingerprint region of spectrum (see Fig. 4(B)), which provided a very nice correspondence between the simulated (middle frame) and the observed (upper frame) spectra.

We found that the transformation of PAT into allyl-isocyanate and phenylazide proceeds with low efficiency. Allowing for $48 \%$ of consumed reagent, only $8 \%$ (i.e. 1/6) is attributed to the reaction Pathway 1. On the other hand, the secondary transformation of phenylazide into ACTH was carried out with relatively high efficiency. Three quarters of the $8 \%$ attributed to this reaction channel were converted into ACHT [(Ph-NNN 2\%) + (ACHT, $6 \%)=8 \%$ ].

Reaction Pathway 2 represented in Fig. 3 corresponds to tetrazole ring-opening leading to allylazide and phenyl-isocyanate as photoproducts. In the experimental spectrum of the photoproducts, the band that appears at $2085.6 \mathrm{~cm}^{-1}$, in close proximity to the corresponding band for phenylazide, is ascribed to the $v \mathrm{~N}=\mathrm{N}^{+}=\mathrm{N}^{-}$antisymmetric stretching vibration of allylazide. The spectrum of phenylisocyanate in solid argon has been reported, ${ }^{12}$ and is easily identifiable because it shows a very intense vibration in the $2290-2260 \mathrm{~cm}^{-1}$ region, due to the $\mathrm{N}=\mathrm{C}=\mathrm{O}$ antisymmetric stretching, that dominates the spectrum. In the spectrum of photoproducts (Fig. 4(A)) this vibration emerges as a doublet at $2280.3 / 2265.3 \mathrm{~cm}^{-1}$ clearly demonstrating the formation of phenyl-isocyanate.

The conversion of PAT into phenyl-isocyanate and allylazide proceeds with relatively high efficiency. Considering the total amount of consumed reagent (48\%), 16\% (i.e. $1 / 3)$ is attributed to the reaction Pathway 2. Moreover, there is no indication that the compounds formed in this channel undergo additional reactions.

Photochemical Pathway 3 in PAT corresponds to a $[3+2]$ pericyclic molecular nitrogen elimination reaction, which leads to formation of a three-membered heterocyclic ring, namely the 1-allyl-2-phenyldiaziridin-3-one (APD) (see Fig. 3). To the best of our knowledge, this species was never described before.

Recently, we have described the photo-induced elimination of molecular nitrogen from 1-phenyl-tetrazolone to give phenyldiaziridin-3-one. ${ }^{12}$ Diaziridinones can be easily identified because their most intense IR band, corresponding to a vibrational mode with dominant contributions from the $\nu \mathrm{C}=\mathrm{O}$ and $\nu \mathrm{C}-\mathrm{N}$ antisymmetric coordinates, occurs in a "clean" region of the spectrum (1900-1850 $\mathrm{cm}^{-1}$ ). In the spectra of the irradiated matrix, the band corresponding to this vibrational mode was clearly identified at $1883.2 \mathrm{~cm}^{-1}$ (Fig. 4(A)), confirming the formation of APD upon PAT photolysis.

Pathway 3 shows a secondary process resulting from conversion of APD into 1-allyl- $1 H$-benzoimidazol-2(3H)-one (ABZ). In the spectra of the photoproducts, the band that appears at $1735.2 \mathrm{~cm}^{-1}$, overlapped with the doublet at $1746.0 / 1740.9 \mathrm{~cm}^{-1}$ due to the $v \mathrm{C}=\mathrm{O}$ antisymmetric stretching vibration of PAT, is attributed to the similar vibration $(\nu \mathrm{C}=\mathrm{O})$ in $\mathrm{ABZ}$ (Fig. $4(\mathrm{~A})$ ). Alternative attributions/photoproducts for the band registered at $1735.2 \mathrm{~cm}^{-1}$ were investigated, but all of them were rapidly discarded. The validity of this attribution is confirmed by a good correspondence between the simulated and the observed spectra of ABZ. Furthermore, it was found that the transformation of PAT in APD plus ABZ occurs with a high efficiency. Allowing for the total amount of consumed reagent (48\%), 24\% is attributed to the reaction Pathway 3.

On the other hand, the secondary transformation of APD into ABZ was carried out with relatively low efficiency (from $24 \%$, only $8 \%$ (i.e. 1/3) was converted in ABZ). APD has a high conformational mobility, but only some part of conformers has a favorable spatial orientation wherein the close proximity of the phenyl and imidazole rings is not affected by the position of allylic fragment and ABZ formation is possible. The restriction of conformational mobility in the matrix can then justify the low yield observed for this secondary transformation.

Interestingly, the allylic chain is maintained unbroken during the irradiation process, indicating that under the experimental conditions used this unit is photochemically stable. Finally, considering the efficiencies of the different reaction channels discussed, a remarkable relationship between their values is detected. The sequence " $0: 8: 16: 24$ " $(\%)$, associated to the efficiencies of Pathways 0 to 3 respectively, can be related as " $0: 1: 2: 3$ " as a perfect and interesting sequence. This is certainly accidental, but still a good example of how Nature can be proud of symbolic beauty!

\section{Conclusions}

The photochemistry of the matrix-isolated 4-allyl-1-phenyltetrazolone (PAT) has been investigated and is now described. The structures of the initial compound and of the photoproducts were determined by infrared spectroscopy, supported by high-level quantum chemical calculations. The DFT(B3LYP)/6$311++\mathrm{G}(\mathrm{d}, \mathrm{p})$ calculations performed in this study predict that, in the gaseous phase, PAT should exist in three different conformers, depending on the position of the ethene group on the allylic chain attached to the tetrazole ring. The potential energy profile for internal rotation of the ethene group was calculated, revealing that the energy barriers separating the three conformers are higher than 
$8 \mathrm{~kJ} \mathrm{~mol}^{-1}$. These barriers are not very high, but they are related with rearrangements of a bulky allyl fragment, which is unlikely to occur in the matrix and thus prevent interconversions between the three conformers. Consequently, the experimental spectrum of PAT closely matches the infrared sum spectrum of the equilibrium mixture of PAT conformers predicted theoretically, pointing to the existence of the three structures in the low-temperature matrix.

The exposure of matrix-isolated PAT to the UV $(\lambda>235 \mathrm{~nm})$ light has been shown to induce three different photochemical pathways: Pathway 1: production of phenylazide and allylisocyanate as primary photoproducts; Pathway 2: production of phenyl-isocyanate and allylazide; and Pathway 3: molecular nitrogen elimination, leading to formation of 1-allyl-2phenyldiaziridin-3-one (APD). The primarily formed phenylazide (Pathway 1) and APD (Pathway 3) are partially converted into 1-aza-1,2,4,6-cycloheptatetraene (ACHT), and 1-allyl-1 $\mathrm{H}$ benzoimidazol-2(3H)-one (ABZ) respectively. Both the vibrational characterisation of APD and the observation of its infrared spectrum were carried out for the first time. The combination of the matrix isolation technique coupled to the FT-IR spectroscopy and aided by theoretical calculations has permitted to identify the photoproducts produced upon photolysis of PAT monomers isolated in an argon matrix.

The non-occurrence (pathway 0) or a partial occurrence (pathway 3 ) in the matrix of the photoreactions involving conformational mobility (and efficient elsewhere in solutions) provided further evidence of the restricted conformational mobility of the studied system in the matrix.

\section{Acknowledgements}

The authors are grateful to Fundação para a Ciência e Tecnologia (FCT) and FEDER [Projects POCI/QUI/59019/2004 and POCI/QUI/58937/2004 and grant SFRH/BD/17945/2004 (L.M.T.F.)] and CONICET (National Research Council, Argentina) for financial support.

\section{References}

1 A. Desarro, D. Ammendola, M. Zappala, S. Grasso and G. B. Desarro, Antimicrob. Agents Chemother, 1995, 39, 232.

2 A. D. Abell and G. J. Foulds, J. Chem. Soc., Perkin Trans. 1, 1997, 2475.

3 Y. Tamura, F. Watanabe, T. Nakatani, K. Yasui, M. Fuji, T. Komurasaki, H. Tsuzuki, R. Maekawa, T. Yoshioka, K. Kawada, K. Sugita and M. Ohtani, J. Med. Chem., 1998, 41, 640.

4 R. J. Mathvink, J. S. Tolman, D. Chitty, M. R. Candelore, M. A. Cascieri, L. F. Colwell, Jr., L. Deng, W. P. Feeney, M. J. Forrest, G. J. Hom, D. E. MacIntyre, L. Tota, M. J. Wyvratt, M. H. Fisher and A. E. Weber, Bioorg. Med. Chem. Lett., 2000, 10, 1971.

5 T. L. Shih, M. R. Candelore, M. A. Cascieri, S.-H. L. Chiu, L. F. Colwell, L. Deng, W. P. Feeney, M. J. Forrest, G. J. Hom, D. E. MacIntyre, R. R. Miller, A. Stearns, C. D. Strader, L. Tota, M. J. Wyvratt, M. H. Fisher and A. E. Weber, Bioorg. Med. Chem. Lett., 1999, 9, 1251.
6 J. Y. L. Chung, G.-J. Ho, M. Chartrain, C. Roberge, D. Zhao, J. Leazer, R. Farr, M. Robbins, K. Emerson, D. J. Mathre, J. M. McNamaraa, D. L. Hughesa, E. J. J. Grabowskia and P. J. Reider, Tetrahedron Lett., 1999, 40, 6739.

7 G. Sandmann, C. Schneider and P. Boger, Z. Naturforsch., C, 1996, 51, 534.

8 G. I. Koldobskii, V. A. Ostrovskii and V. S. Poplavskii, Khim. Geterotsiklicheskikh Soedin., 1981, 1299.

9 C. Zhao-Xu and X. Heming, Int. J. Quantum Chem., 2000, 79, 350.

10 G. Maier, J. Eckwert, A. Bothur, H. P. Reisenauer and C. Schmidt, Liebigs Ann., 1996, 1041.

11 A. Awadallah, K. Kowski and P. Rademacher, J. Heterocycl. Chem., 1997, 34, 113.

12 A. Gómez-Zavaglia, I. D. Reva, L. Frija, M. L. Cristiano and R. Fausto, J. Photochem. Photobiol., A, 2006, 179, 243.

13 A. Gómez-Zavaglia, I. D. Reva, L. Frija, M. L. Cristiano and R. Fausto, J. Mol. Struct., 2006, 786, 182.

14 A. Gómez-Zavaglia, I. D. Reva, L. Frija, M. L. S. Cristiano and R. Fausto, J. Photochem. Photobiol., A, 2006, 180, 175.

15 A. Gómez-Zavaglia, I. D. Reva, L. Frija, M. L. Cristiano and R. Fausto, J. Phys. Chem. A, 2005, 109, 7967.

16 L. M. T. Frija, I. V. Khmelinskii and M. L. S. Cristiano, J. Org. Chem., 2006, 71, 3583 .

17 L. M. T. Frija, I. V. Khmelinskii and M. L. S. Cristiano, Tetrahedron Lett., 2005, 46, 6757.

18 A. Kumar and D. C. Dittmer, J. Org. Chem., 1994, 59, 4760.

19 W. Adam, K. Peters and M. Renz, Angew. Chem., Int. Ed. Engl., 1994, 33, 1107.

20 M. L. S. Cristiano and R. A. W. Johnstone, J. Chem. Soc., Perkin Trans., 1997, 2, 489.

21 M. L. S. Cristiano and R. A. W. Johnstone, J. Chem. Res., Synop., 1997, 164.

22 I. D. Reva, S. G. Stepanian, L. Adamowicz and R. Fausto, J. Phys. Chem. A, 2001, 105, 4773 .

23 A. D. Becke, Phys. Rev. A, 1988, 38, 3098.

24 C. T. Lee, W. T. Yang and R. G. Parr, Phys. Rev. B, 1988, 37, 785.

25 P. Csaszar and P. Pulay, J. Mol. Struct., 1984, 114, 31.

26 J. H. Schachtschneider, Technical Report, Shell Development Co., Emeryville, CA, 1969.

27 M. J. Frisch, G. W. Trucks, H. B. Schlegel, G. E. Scuseria, M. A. Robb, J. R. Cheeseman, J. A. Montgomery, Jr., T. Vreven, K. N. Kudin, J. C. Burant, J. M. Millam, S. S. Iyengar, J. Tomasi, V. Barone, B. Mennucci, M. Cossi, G. Scalmani, N. Rega, G. A. Petersson, H. Nakatsuji, M. Hada, M. Ehara, K. Toyota, R. Fukuda, J. Hasegawa, M. Ishida, T. Nakajima, Y. Honda, O. Kitao, H. Nakai, M. Klene, X. Li, J. E. Knox, H. P. Hratchian, J. B. Cross, V. Bakken, C. Adamo, J. Jaramillo, R. Gomperts, R. E. Stratmann, O. Yazyev, A. J. Austin, R. Cammi, C. Pomelli, J. Ochterski, P. Y. Ayala, K. Morokuma, G. A. Voth, P. Salvador, J. J. Dannenberg, V. G. Zakrzewski, S. Dapprich, A. D. Daniels, M. C. Strain, O. Farkas, D. K. Malick, A. D. Rabuck, K. Raghavachari, J. B. Foresman, J. V. Ortiz, Q. Cui, A. G. Baboul, S. Clifford, J. Cioslowski, B. B. Stefanov, G. Liu, A. Liashenko, P. Piskorz, I. Komaromi, R. L. Martin, D. J. Fox, T. Keith, M. A. Al-Laham, C. Y. Peng, A. Nanayakkara, M. Challacombe, P. M. W. Gill, B. G. Johnson, W. Chen, M. W. Wong, C. Gonzalez and J. A. Pople, GAUSSIAN 03 (Revision C.02), Gaussian, Inc., Wallingford, CT, 2004.

28 I. D. Reva, S. G. Stepanian, L. Adamowicz and R. Fausto, Chem. Phys. Lett., 2003, 374, 631.

29 H. Quast and U. Nahr, Chem. Ber.-/Recl., 1985, 118, 526.

30 I. R. Dunkin, C. J. Shields and H. Quast, Tetrahedron, 1989, 45, 259.

31 H. Quast, A. Fuss and U. Nahr, Chem. Ber.-/Recl., 1985, 118, 2164.

32 H. Quast, Heterocycles, 1980, 14, 1677.

33 H. Quast and L. Bieber, Chem. Ber.-/Recl., 1981, 114, 3253.

34 K. A. Jensen, M. Due and A. Holm, Acta Chem. Scand., 1965, 19, 438.

35 M. D. Halls and H. B. Schlegel, J. Chem. Phys., 1998, 109, 10587. 